\title{
Study of ultrasound-modulated optical tomography in biological tissue with parallel detection
}

\author{
Gang $\mathrm{Yao}^{\prime}$, and Lihong V. Wang ${ }^{1,2}$
}

\begin{abstract}
An ultrasonic beam was focused into a biological tissue sample to modulate the laser light passing through the ultrasonic beam inside the tissue. The speckle field formed by the transmitted laser light was detected by a CCD camera with the source-synchronous-illumination lock-in technique. The ultrasound-modulated laser light reflects the local optical and mechanical properties within the ultrasonic beam and can be used for tomographic imaging of the tissue. Spatial resolution along the ultrasonic axis was achieved by sweeping the ultrasonic frequency. Two-dimensional images of biological tissue were successfully obtained with both single frequency modulation and frequency-swept modulation. Threedimensional images could be acquired as well in principle.
\end{abstract}

Key words - optical imaging, ultrasound modulation, biological tissue.

\section{INTRODUCTORY}

A number of optical imaging techniques ${ }^{1}$ have been studied for imaging in biological tissues. The key challenge is to overcome the strong scattering of light in biological tissues. Besides purely optical imaging techniques, techniques combining optical techniques and ultrasonic techniques have also been explored because ultrasonic waves scatter much less in biological tissues than optical waves and can directly furnish localization information for imaging. Ultrasoundmodulated optical technique $e^{2-5}$ is one of such hybrid methods. Marks et al. ${ }^{2}$ investigated the possibility of tagging light with ultrasound. Wang et al $^{3}$ developed ultrasound-modulated optical tomography and obtained images in tissue-simulating phantoms. A single-detector scheme was usually used in these early studies. Recently, Leveque et al. ${ }^{6}$ developed a parallel speckle detection scheme using a source-synchronized lock-in technique, in which a CCD camera worked as a detector array. The signal-to-noise ratio (SNR) can be greatly improved by averaging the signals from all of the CCD pixels. They obtained one-dimensional (1D) images of buried object in real biological tissue (turkey breast).

However, in those experiments, the spatial resolution along the ultrasonic axis is rather poor because the imaging signal consists of contributions from a relatively long zone along the ultrasonic column. Wang and $\mathrm{Ku}^{7}$ developed a technique called frequency-swept ultrasound-modulated

\footnotetext{
1 Optical Imaging Laboratory, Biomedical Engineering Program,

Texas A\&M University, College Station, TX 77843-3120

2

To whom correspondence should be addressed.

Tel: (409) 847-9040, Fax: (409) 845-4450, Email: lwang@tamu.edu,

URL: http://oilab.tamu.edu
}

optical tomography to achieve spatial resolution along the ultrasonic axis. In their experiment, one frequency-sweep function (chirp) excited the ultrasonic transducer while another chirp modulated the gain of the optical detector. When the heterodyne signal from the optical detector was Fourier transformed, the signal from a specific spatial location was converted into a specific frequency component. However, because of the limited SNR of the single-detector scheme, this technique was demonstrated only for ballistic imaging.

We further investigated imaging of biological tissue based on parallel detection of ultrasound-modulated laser speckles with the synchronous-illumination lock-in technique. Twodimensional images of biological tissues with a laser beam of oblique incidence were obtained, which indicated that this technique was not dependent on purely ballistic photons. In order to achieve spatial resolution along the ultrasonic axis, we extended the frequency-swept modulation technique in this study using parallel detection. The laser and the ultrasonic transducer were both modulated with chirp functions. Imaging along the ultrasonic axis was obtained by electronically scanning the time delay between these two functions. We demonstrated this technique by acquiring 2D images of biological tissues with buried objects.

\section{EXPERIMENTAL SETUP}

In ultrasound-modulated optical tomography, the transmitted light consists of two parts: the $\mathrm{AC}$ photons that are modulated by the ultrasonic wave; and the DC background photons that are not modulated. The AC photons contribute to the signal, while the DC photons contribute to the shot noise, which is the main noise source in the experiments. The AC signal intensity depends on two factors: the number of modulated photons and their modulation depth. The modulation depth, usually less than $10 \%$ in the experiments, is related to the intensity of the ultrasonic wave and the modulation mechanisms. ${ }^{8,9}$ Only the photons passing through the ultrasonic column could be modulated. The number of modulated photons is related to the optical properties of the scattering media and the size of the modulation column that determines the spatial resolution. Therefore, the quantity AC/DC reflects the local optical and mechanical properties within the ultrasonic beam and can be used for tomographic imaging of the tissue. 
Proceedings of the $22^{\text {nd }}$ Annual EMBS International Conference, July 23-28, 2000, Chicago IL.

A speckle pattern is formed after the light passing through the highly scattering biological tissue (Fig. 1). Because the phase of each single speckle is randomly distributed between $(-p,+p)$, averaging signals from large quantity of speckles

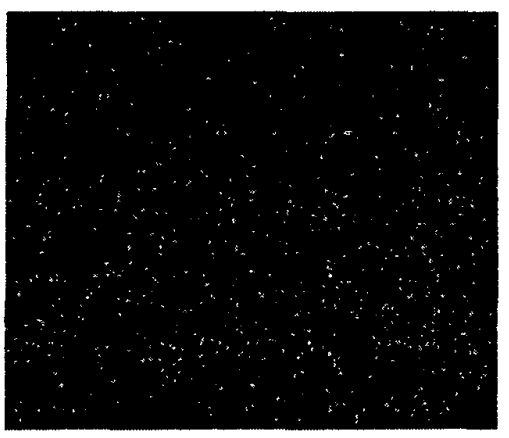

Fig.1. A typical speckle pattern generated by laser passing through a chicken breast tissue.

decreases $\mathrm{AC} / \mathrm{DC}{ }^{4}$ In other words, a single speckle detection scheme is necessary to achieve high signal intensity in the measurement. Considering the usually very small size of the laser speckle, CCD camera is appropriate in this application. Each CCD pixel can act as a single detector. And because the large quantity of $\mathrm{CCD}$ pixels, we can greatly improve the SNR ratio by averaging the signals from all the pixels. To overcome the low response frequency of CCD camera, source-synchronous-illumination lock-in technique ${ }^{11}$ was implemented in the experiment by modulating the laser source at the signal frequency.

The experimental setup is shown in Fig. 2. A coordinate system was set up for reference. The $Z$ axis was along the optical axis pointing to the CCD camera. The $Y$ axis was on the acoustic axis pointing from the ultrasonic transducer to the sample. The $X$ axis was perpendicular to both the acoustic and optical axes:

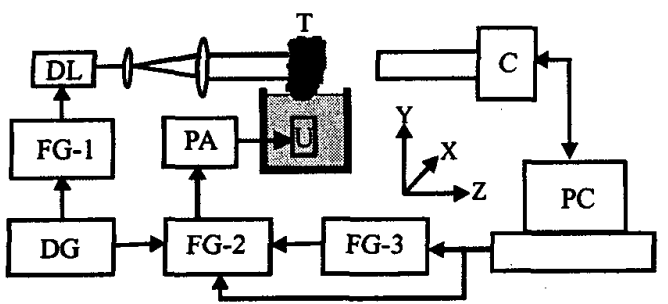

Fig.2. Schematic of the experimental setup. DL: diode laser; $\mathrm{C}$ CCD camera; U: ultrasonic transducer; FG-1, FG-2 and FG-3: function generators; DG: delay generator; PA: power amplifier; $T$ : tissue sample.

The ultrasonic waves were generated by a focused ultrasonic transducer (Panametrics, V314) with a 2.54-cm focal length in water and a $1-\mathrm{MHz}$ central response frequency. The peak pressure at the focus was about $2 \times 10^{5}$ $\mathrm{Pa}$, well below the damage threshold for biological tissues. ${ }^{10}$ The laser beam from a diode laser (Melles Griot, 56IMS667, 690-nm wavelength) was expanded and projected directly onto the tissue sample. The average power of the modulated laser beam was $12 \mathrm{~mW}$. The coherence length of the modulated laser was measured to be $\sim 7 \mathrm{~cm}$. The tissue sample was partially immersed in the water for good acoustic coupling. The speckle pattern generate by the transmitted light was detected by a high-speed digital CCD camera (Dalsa CA-D1-0256T). The three function generators (Stanford Research Systems, DS345) FG-1, FG-2 and FG-3 shared the same time base to ensure synchronization.

The tissue samples used in the experiments were skinless chicken breast tissues bought from a supermarket. The objects buried in the tissue were made firom soft rubber, which has good acoustic coupling with tissue and has little acoustic absorption.

\subsection{Single frequency modulation}

In single frequency modulation, FG-1 and FG-2 generated single frequency $(1 \mathrm{MHz})$ sinusoidal waves to modulate the diode laser and to excite the ultrasonic transducer, respectively. FG-3 was bypassed in the experiments. The signal in a single CCD pixel can be represented as: ${ }^{11}$

$$
\left.I_{i} \quad I_{b}+I_{n, 1} \cos \phi+\phi_{i}\right),
$$

where $I_{b}$ is the background intensity, $I_{m}$ is the signal intensity related to the ultrasound-modulated component, $\phi$ is the randomly distributed initial phase of the speckle, and $\phi_{i}$ is the initial phase of the sinusoidal wave from FG-2. The quantity $M=I_{m} / I_{b}$ is related to the modulation depth, which reflects the local optical and ultrasonic properties. The initial phase $\phi_{i}$ of this sinusoidal wave was set sequentially to $0,90,180$, and 270 . The corresponding four frames of CCD images were acquired to calculate $M$ as follows:

$$
\left.\left.M=\frac{1}{2 I_{b}} \mid \psi_{90^{\circ}}-I_{270^{\circ}}\right)^{2}+\psi_{0^{\circ}}-I_{180^{\circ}}\right)\left.^{2}\right|^{\frac{1}{2}} \text {. }
$$

The above calculation was performed for each pixel of the CCD camera. A total of $256 \times 256$-pixel data were then averaged to produce a single data point for the image. We averaged 50 such measurements to further improve the SNR.

In single frequency modulation scheme, each imaging data point is essentially an integration of signals along the ultrasonic column. The spatial resolution along the ultrasonic axis cannot be obtained because the focal depth of the ultrasonic transducer is about $2 \mathrm{~cm}$ in the current setup.

\subsection{Frequency-swept modulation}

We implemented frequency-swept modulation to obtain spatial resolution along ultrasonic axis. FG-1 and FG-2 generated chirp functions to modulate the diode laser and to excite the ultrasonic transducer, respectively. The delay/pulse generator DG controlled the time delay between the trigger signals to FG-1 and FG-2. If the signal from FG-2 were not amplitude-modulated by FG-3, the frequency of the heterodyne signal from location $y$ along the ultrasonic axis $(Y$ axis) would be: ${ }^{7}$

$$
f(y, \tau)=b \tau-\frac{y}{v_{s}},
$$

where $b$ is the frequency sweep rate, $v_{s}$ is the ultrasonic velocity, and $\tau$ is the time delay between the two chirps from FG-2 and FG-1. To implement the source-synchronized lock- 
Proceedings of the $22^{\text {nd }}$ Annual EMBS International Conference, July 23-28, 2000, Chicago IL.

in technique, we used FG-3 to produce a sinusoidal wave with a frequency equal to $f(y, \tau)$, which modulated the amplitude of the chirp function generated by FG-2. The initial phase $\phi_{i}$ of this sinusoidal wave from FG-3 was set sequentially to $0^{\circ}$, $90^{\circ}, 180^{\circ}$, and $270^{\circ}$ and the modulation depth was calculated according to Eq. (2).

In the experiments, the time delay $\tau$ was scanned while the lock-in frequency from FG-3 was set to a fixed value $f_{0}$. From Eq. 3, the ultrasound-modulated light from a specific spatial location $y_{0}$ corresponding to the heterodyne frequency $f_{0}$ was detected. The ultrasound-modulated light from the other spatial locations had different frequencies and hence was rejected by the CCD camera. Therefore, one-dimensional images along the ultrasonic axis could be obtained by electronically scanning the time delay $\tau$. To obtain 2D tomographic images, the ultrasonic transducer was mechanically scanned by a translation stage along the $X$ axis.

\section{EXPERIMENTAL RESULTS}

\subsection{Single frequency modulation}

Figure 3(a) shows a 2D ultrasound-modulated optical image obtained from $1.5-\mathrm{cm}$ thick chicken breast tissue with the light beam illuminating the sample obliquely at $15^{\circ}$ to the $Z$ axis. The sizes of the two buried objects were roughly: $2.5 \times$ $2.8 \times 7.1 \mathrm{~mm}^{3}$ and $2.3 \times 2.7 \times 6.9 \mathrm{~mm}^{3}$, respectively. In the experiments, the tissue sample was scanned along the $X$ axis, and the ultrasonic transducer was scanned along the optical axis ( $Z$ axis). The scanning step size is $1 \mathrm{~mm}$. The relative lateral positions of the incident light with the ultrasonic
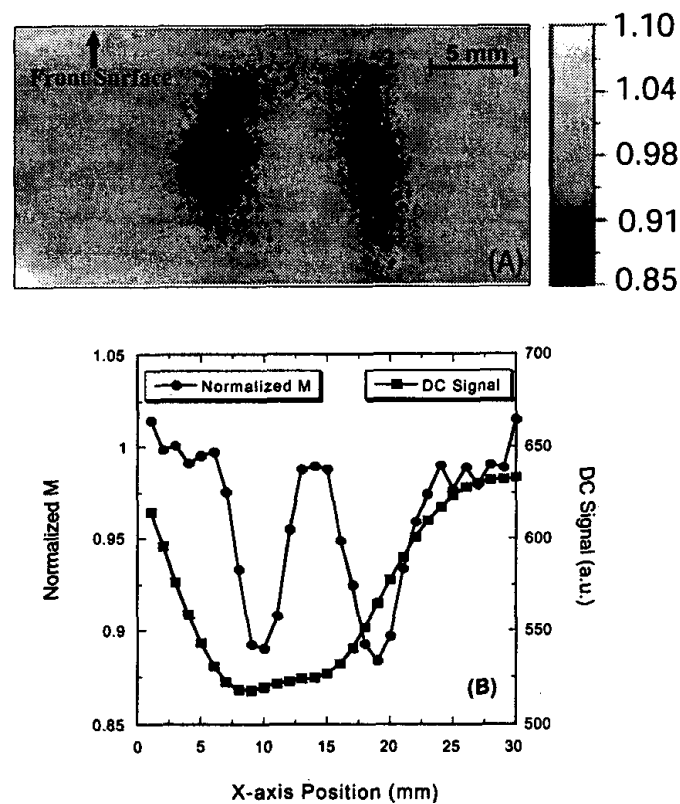

Fig.3. Experimental images of $1.5-\mathrm{cm}$ thick chicken breast tissue with a laser beam obliquely incident at $15^{\circ}$. (a) $2 \mathrm{D}$ cross-sectional image; (b) ID image along the $X$ axis at the center of the sample. The front surface faced the laser beam. column and the exit aperture were fixed in the scanning process. The input diameter of the collection tube was $1.6 \mathrm{~cm}$, and the length from the input end to the CCD chip was $\sim 38$ $\mathrm{cm}$. Therefore, the speckle size was $\sim 16 \mu \mathrm{m}$, which was equal to the CCD pixel size. The data in the image was normalized to the background value, and the 2D image was low-pass filtered. The two objects are clearly visible in the $2 \mathrm{D}$ image. Figure $3(\mathrm{~b})$ shows a 1D plot corresponding to the scan line along the $X$ axis at the center of the tissue. From Fig. 3(b), the two objects are not discriminable in the DC-transmittance data, while they are sharply observed in the modulated data. The spatial resolution is approximately $2 \mathrm{~mm}$, which is determined by the focal size of the $1-\mathrm{MHz}$ ultrasonic transducer used in the experiments.

As previously measured, ${ }^{12}$ the reduced scattering coefficient of the chicken breast tissue is about $2 \mathrm{~cm}^{-1}$, and the absorption coefficient is about $0.1 \mathrm{~cm}^{-1}$ at $\sim 700 \mathrm{~nm}$. Therefore, the penetration depth is $\sim 1 \mathrm{~cm}$. At this penetration depth, ballistic photons are not totally undetectable. However, any ballistic photons, if existed, would have missed the detector at oblique incidence of $15^{\circ}$. Therefore purely ballistic photons are not the major contributors to the signal in our experiments and ultrasound-modulated optical tomography mainly depends on diffuse light.

\subsection{Frequency-swept modulation}

Figure 4 shows a 2D image obtained from a $1.2-\mathrm{cm}$ thick chicken breast tissue with a buried object. The size of the object was $4.0 \mathrm{~mm} \times 2.7 \mathrm{~mm} \times 6.2 \mathrm{~mm}$ along the $X, Y$, and $Z$ axes, respectively. The step size of the electronic scan along the $Y$ axis was $1.0 \mu \mathrm{s}$, corresponding to $1.58 \mathrm{~mm}$ in the tissue. The step size of the mechanical scan along the $X$ axis was 1.5



Fig.4. Experimental 2D image of 1.2-cm thick chicken breast tissue containing a buried object.

$\mathrm{mm}$. The raw $1 \mathrm{D}$ images along the $Y$ axis were normalized by the result obtained from uniform background tissue so as to compensate for the different detection efficiencies caused by the small detection area $(4 \mathrm{~mm} \times 4 \mathrm{~mm})$ of the CCD camera and the ultrasonic pressure distribution along the ultrasonic axis. In the image shown in Fig. 4, the buried object is clearly visible in the background. The image resolution along the $X$ axis is $\sim 2 \mathrm{~mm}$, which is determined by the $2-\mathrm{mm}$ focal diameter of the ultrasonic transducer used in the experiments.

The spatial resolution along the ultrasonic axis ( $Y$ axis) $\Delta y$ is determined by the frequency span $\Delta f$ of the chirp function 


\section{Proceedings of the $22^{\text {nd }}$ Annual EMBS International Conference, July 23-28, 2000, Chicago IL.}

and the ultrasonic velocity $v_{s}$ as follows: $\Delta y \approx v_{s} / \Delta f .^{7}$ In the experiments, $v_{s} \approx 1,580 \mathrm{~m} / \mathrm{s}$, and $\Delta f=400 \mathrm{kHz}$, which yields $\Delta y \approx 4 \mathrm{~mm}$. The spatial resolution along the ultrasonic axis can be scaled by changing the frequency span $\Delta f$. To demonstrate this scalability, we acquired two 1D images with frequency spans $\Delta f=400 \mathrm{kHz}$ and $\Delta f=800 \mathrm{kHz}$, corresponding to spatial resolution of $4 \mathrm{~mm}$ and $2 \mathrm{~mm}$, respectively [Fig. 5]. The buried object had a thickness of 3

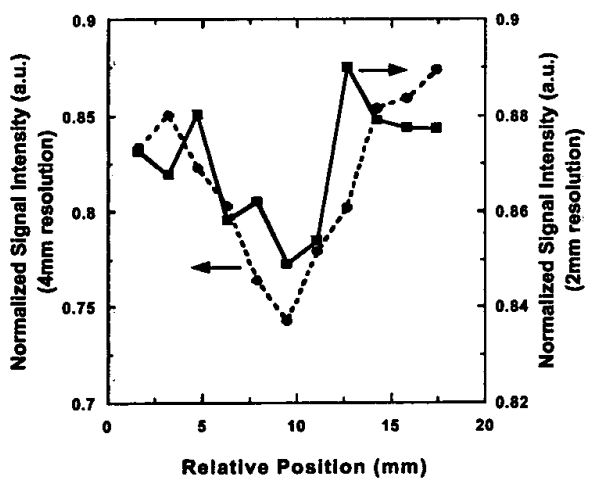

Fig.5. 1D images of 1.2-cm thick chicken breast tissue with spatial resolution of $4 \mathrm{~mm}$ and $2 \mathrm{~mm}$ along the ultrasonic axis.

$\mathrm{mm}$ along the $Y$ axis. As shown in the results, the 1D image obtained with $\Delta f=800 \mathrm{kHz}$ has sharper edges than the image obtained with $\Delta f=400 \mathrm{kHz}$. However, the intensity of the signal with the better spatial resolution is less because the spatial volume that contributes to the signal is reduced.

\section{CONCLUSION}

The ultrasound-modulated optical tomography has the potential for early detection of breast cancers, because it combines the contrast advantage of purely optical imaging and the resolution advantage of purely ultrasonic imaging and optical properties are related to tissue constituents and their molecular conformations. By using a low power laser, we obtained $2 \mathrm{D}$ images of chicken breast tissue up to $1.5 \mathrm{~cm}$ thick.

Due to the small area of the CCD chip, only a small portion of the transmitted light was utilized in the image acquisition. In order to maintain a high enough SNR, the CCD camera needs to receive enough photons by using a long exposure time. In our experiments, the single-frame exposure time was $\sim 150 \mathrm{~ms}$. However, besides slowing down the data acquisition, the long exposure time may cause the SNR to suffer from decorrelation of the speckle field. Obviously, this problem could be overcome by simply increasing the incident laser power. The average power density in our experiments is $<25 \mathrm{~mW} / \mathrm{cm}^{2}$, which can be raised to $200 \mathrm{~mW} / \mathrm{cm}^{2}$, limited by the ANSI standards for 690 -nm laser light. In addition, the SNR could also be improved by using a detector with a higher dynamic range because the modulated signal is currently very small compared with the background. It is capable to image thicker sample by these improvements.

In addition, by combining the frequency-swept ultrasound-modulation technique with the parallel-detection scheme, we obtained 2D images of chicken breast tissue containing buried objects with spatial resolution of $\sim 4 \mathrm{~mm}$ along the ultrasonic axis. The lateral spatial resolution was $\sim 2$ $\mathrm{mm}$ and was determined by the focal width of the ultrasonic transducer. This technique can be directly applied to acquire three-dimensional images in turbid media.

ACKNOWLEDGMENT This project was sponsored in part by the National Institutes of Health grants R29 CA68562, R01 CA71980, and R21 CA83760 and by the National Science Foudation grant BES-9734491.

\section{References}

1. J. G. Fujimoto and M. S. Patterson, eds., Advances in Optical Imaging and Photon Migration (Optical Society of America, Washington, DC 1998).

2. F. A. Marks, H. W. Tomlinson, and G. W. Brooksby, "Comprehensive approach to breast cancer detection using light: photon localization by ultrasound modulation and tissue characterization by spectral discrimination," in Photon Migration and Imaging in Random Media and Tissue, B. Chance and R. R. Alfano, eds., Proc. SPIE 1888, 500510(1993).

3. L.-H. Wang, S. L. Jacques, and X. Zhao, "Continues-wave ultrasonic modulation of scattered laser light to image objects in turbid media," Opt. Lett. 20,629 (1995).

4. L.-H. Wang and X. Zhao, "Ultrasound-modulated optical tomography of absorbing objects buried in dense tissue-simulating turbid media," Appl. Opt. 36, 7277 (1997).

5. M. Kempe, M. Larionov, D. Zaslavsky, and A. Z. Genack, "Acoustooptic tomography with multiple scattered light," J. Opt. Soc. Am. 14, 1151 (1997).

6. S. Leveque, A. C. Boccara, M. Lebec, and H. Saint-Jalmes, "Ultrasonic tagging of photon paths in scattering media: parallel speckle modulation processing,"Opt. Lett. 24, 181 (1999).

7. L.-H. Wang, and G. Ku, "Frequency-swept ultrasound-modulated optical tomography of scattering media," Opt. Lett. 23, 975 (1998).

8. W. Leutz and G. Maret, "Ultrasonis modulation of multiply scattered light," Physica B 204, 14-19 (1995).

9. L.-H. Wang, "Ultrasonic modulation of scattered light in turbid media and a potential novel tomography in biomedicine," Photochem. Photobiol. 67, 41-49 (1998).

10. T. A. Whittingham, "The safety of ultrasound," Imaging 6, 33 (1994).

11. P. Gleyzes, F. Guernet and A. C. Bciccara, "Picometric profilometry. II. Multidetector approach and multiplexed lock-in detection," J. Opt. 26, 251-265(1995).

12. G. Marquez, L.-H. Wang, S.-P. Lin, J. A. Schwartz, and S. L. Thomsen, "Anisotropy in the absorption and scattering spectra of chicken breast tissue," Appl. Opt. 37, 798-805 (199:8). 